\section{The Largest Possible Microscope}

Sterling P. Newberry, Consultant

In this day of resistance toward big science projects it may be futile to worry about how to produce the largest possible microscope. But since it doesn't cost anything to day dream, and times may change, it is fun to consider superlative enterprises.

One must grant that Ultra High Voltage Electron Microscopes are much larger than we wish they were, and one wonders if there is a contest afoot to see which AEM can have the largest foot print, but a new possibility has arisen which, seriously, could dwarf them all - yet have some possible use.

I am referring to the discovery of an example of a gravitation lens for ordinary light. Now the distinguishing characteristic of a microscope from a telescope is that the object eyepiece and the object near the focal length of the lens. Thus, in principle, if one aligned an eyepiece toward the center of the gravitational lens he could see an image of objects passing near the principal focus of that lens.

is the idea utter nonsense, I think not. The resolution would not be great, but the objects would be quite large in comparison to the resolution. The apparent high symmetry of the gravitational lens suggests that it would be analogous to using a simple spherical glass lens as Van Leeuwenhock had to do. The advantage we have over Van Leeuwenhock is that today we can correct out most of the geometrical aberrations electronically. Furthermore we would use low noise, large area photon detectors instead of a simple eyepiece. Perhaps the orbiting telescope "Galileo" can be made either to behave as an eyepiece or has sensors which are not obscured by a lens and could provide a test of the idea in the ideal conditions of space without requiring a separate mission to test it. Maybe we could even test it here on earth with detectors mounted on existing radio telescope antennas, but at first blush, that approach does not look very plausible. Probably our best plan is to design a sensor array to be placed on Galileo's frame at our next routine maintenance rendezvous with it.

\section{Image Archiving Made Simple, Fast \& Complete!}

\section{Advanced Imaging Concepts, Inc., provider of image analysis} systems, offers a user-definable solution to intelligent image management and archiving. Featuring exceptional ease of use, Windows based Image Central enables you to rapidly retrieve, sort, query, and view database records (images with data). Image Central works with live images or those stored in any of several formats, such as TIFF, TGA, PCX, GIF, and BMP.
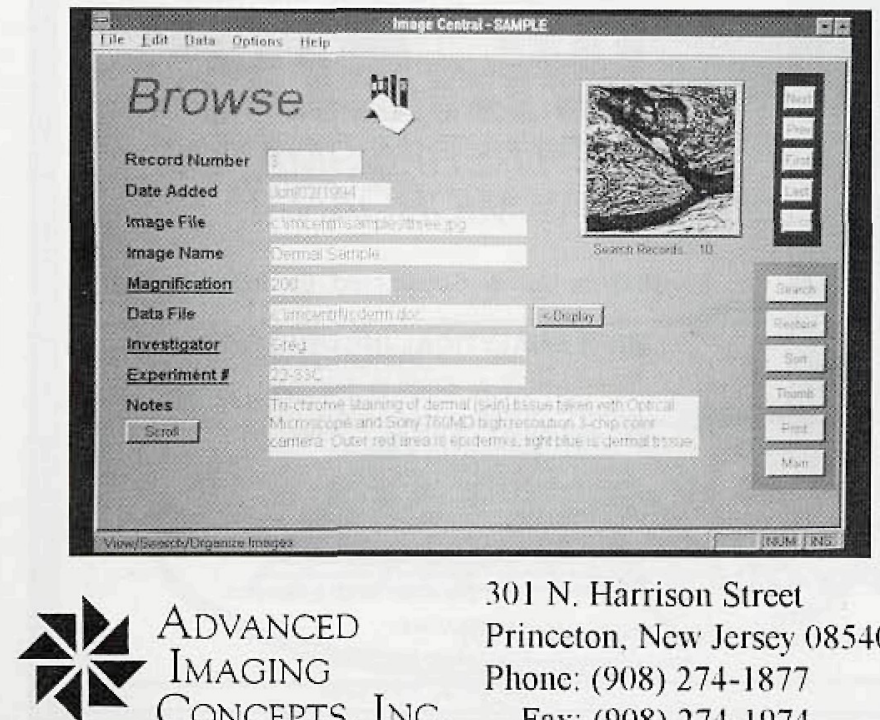

ADVANCED IMAGING CONCEPTS, INC.

301 N. Harrison Street

Princeton. New Jersey 08540

Phone: (908) 274-1877

Fax: (908) 274-1974

\section{ISO 9002 in the Microscopy Laboratory}

\section{A.J. Terry, ORTECH Corporation}

Many laboratories today wish to assure their clients of the implementation of a sound quality system. The International Standards Organization (ISO) has the 9000 series of quality systems which is recognized globally. This article specifically addresses issues to be considered for a good quality system in a modern service oriented microscopy laboratory.

The quality system is devised to attain certain objectives which are consistent throughout the company, whether they be research and development, marketing and sales, supply of a service, procurement, shipping, etc. Each company devises a quality systern specific to their business and that serves their own and their client's needs. Some of these objectives may be the following: assurance of cost effective operations; prevention of errors in all aspects of the operation; assurance to the client that the objectives, deliverables and time frame are understood; prevention of delays; increase of productivity, a commitment to meeting the client's quality assurance requirements.

In order to ensure trackability of each sample and product which may be used in a specific project, a unique identifying number is assigned in a consistent manner according to a standard operating procedure (SOP) detailed by the microscopy laboratory. Each laboratory assigns sample numbers in a similar fashion which facilitates tracking back to the receiving laboratory, whether the sample be submitted by hand, mail, courier, to receiving, etc. . Batches of samples, sub-samples and samples produced in the lab are also identified in a unique way. Received along with the samples, whether intermnally or externally, is authorization to proceed with the proposed work. This includes a description of the work to be performed, the time frame for the project and associated costs, i.e the "Quality Plan".

Separate lab books are used for different types of analyses and projects. Again, these are trackable to specific samples and reports, which (as in the case of samples) are also given unique identification numbers in accordance with the quality system procedures. In addition, the instrumentation used in the work and its calibration status are recorded in the laboratory books. Measuring instruments are identified by means of a unique reference number and specific calibration dates are assigned, appropriate to the instrumentation and frequency of use.

Examples of measuring instruments in the microscopy laboratory are: the scanning electron microscope (SEM); the energy dispersive $X$-ray (EDX) spectrometer; the measurement programs in the image analysis software; the $X$-ray diffractometer; the transmission electron microscope and associated EDX spectrometer and selected area electron diffractometer (SAED); the various light microscopes; stage micrometers; a taceable National Institute of Standards and Technology (NIST) stage micrometer scale for calibration of the SEM; air sampling pumps, etc. Calibration checks are made for some of the day to day operations which require more rigorous calibration at specified intervals. Reference standards are on hand for confirmation of properties to be tested, e.g. NIST asbestos Standard Reference Material (SRM), various minerals and fibres, aerosol monitor filters with quantified concentrations of airborne fibres, bulk building materials with quantified concentrations of asbestos, metals with known concentrations of various elements, etc.

All standard test procedures are originals that have been ordered through the corporate business resource centre which monitors the publication dates to ensure that obsolete methodology is not used. The standard test and calibration procedures, as well as in-house testing and calibration procedures are documented in SOPs and make reference to measuring instrument reference numbers and traceable standards, where appropriate.

A log is also kept for disposal of samples and records associated with projects. Samples are disposed on in a safe manner which conforms to existing regulations or are returned to the client if requested. Records are kept for a specified minimum period of time.

Third party certification with, for example, other ISO 9000 registered organizations demonstrates internal commitment and the implementation of a quality system adequate for the services offered. This reduces the costs for multiple assessments by multiple clients and assures a process for continuous improvement, due to the nature of the quality system structure. 